\title{
Commentary on "Timbre as an Elusive Component of Imagery for Music," by Freya Bailes
}

\author{
ANDREA R. HALPERN \\ Bucknell University
}

\begin{abstract}
The study of musical timbre by Bailes (2007) raises important questions concerning the relative ease of imaging complex perceptual attributes such as timbre, compared to more unidimensional attributes. I also raise the issue of individual differences in auditory imagery ability, especially for timbre.
\end{abstract}

Submitted 2006 November 21; Accepted 2006 November 25

KEYWORDS: timbre, mental imagery, perception, memory, methodology, individual differences

TIMBRE has a somewhat paradoxical status in music psychology. The literature on timbre perception and memory is small compared to music cognition studies on dimensions such as pitch and rhythm. On the one hand, we find that people can discriminate and classify timbre reasonably well. This is demonstrated in the pretests of the article by Bailes (2007) as well as in numerous studies showing that musical timbres are mentally represented in a regular manner, some of which Bailes cites. And of course, composers select and then change timbres to convey various musical messages.

On the other hand, timbre perception is influenced by pitch (Krumhansl \& Iverson, 1992), suggesting it is not an independent dimension. It is also not an easy dimension to remember: I have numerous experiments in my bottom drawer showing a failure of participants to remember what instrument played a given tune during a study list. Even indirect demonstrations of timbre memory can be hard to obtain. In a musical stem completion task, I found no effect of timbre change on ability to remember explicitly, by singing the requested note, what note came next after the first few notes of a studied tune was played. Nor did I find a detrimental effect of timbre change on the implicit task of producing a note that "sounded good," where a higher rate of correct completions on studied vs. unstudied items would indicate preserved implicit memory (Warker \& Halpern, 2005).

Matching a timbre to an entire tune may be a challenging binding task. The few studies in timbre imagery have typically asked participants to imagine single notes. Crowder (1989) found interference effects from timbre in a pitch-matching task when the imagined tone of one pitch did not match the actual tone of another pitch (also showing the pitch-timbre interdependence referred to above). Pitt and Crowder (1992) likewise used single tones to investigate spectral vs. dynamic aspects of timbre imagery. And Halpern et al. (2004) asked participants to imagine single tones of various instruments for a similarity rating task. All of these studies used single tones for a good reason: to insure that the timbre in question was active in working memory at the time the particular discrete judgment was made. And they also serve to show that people can (in the case of similarity judgments) or must (in the case of interference effects) reactivate what appear to be perceptual-like representations of timbre.

Bailes points out that it would also be useful to examine timbre imagery for more extended musical passages, although it is perhaps unduly strong to say that the previous studies cannot be generalized at all to the extended situation. The current study has some similarities to previous ones, in that some comparison is made between perceptual and imaginal judgments. Also congruent with previous studies, judgments made when part of the stimulus is imagined led to lower performance levels than when the stimulus is heard. As we pointed out in our 2004 article, participants in Crowder's (1989) study were about $500 \mathrm{~ms}$ slower to carry out pitch matching when the first note of a pair was imagined rather than heard, and in one of our earlier studies, average accuracy in a pitch comparison task within a song fell from $85 \%$ in a heard version to $73 \%$ when the song was imagined (Zatorre \& Halpen, 1993). In the 2004 study, the multidimensional scaling solution for imagined instruments was less regular than that for perceived 
instruments (although the fit of the model was equally good in both cases). All of these studies converge on showing that reactivated information provides some information, but not all the information necessary to make timbre-related judgments.

Bailes takes a different tack in both the method and conceptualization of imagery in this article. In both studies she asks participants to memorize a piece of music. In the first study, she then asks them to compare a sounded note pair against a memory of that note pair for a same-different judgment on timbre, and manipulates whether the rest of the memorized piece is sounded or not. So two things are really going on here: a) matching a timbre representation to that supposedly in long-term memory and b) using the sounded intervening notes as a memory aid, insofar that it is likely that the immediately preceding note pair acts as a cue (or contrast) to the next, to-be-judged note pair. In the second study, the timbre judgment is a more subtle change, but still involves, or could involve, the two aspects described above: memory for the learned timbre per se, and making use of the contrast from the information immediately preceding the tobe-judged phrase. Because results of the first study are potentially open to alternative explanations involving practice effects, I prefer to concentrate on the second study.

The main result is that time to make the judgment (I assume this includes all trials, as we are not told that RT is only to correct trials) decreases from perception to imagery to control. This latter condition took place before the piece was learned and was meant to capture pure discrimination. Accuracy showed the inverse pattern in one piece and did not vary for the other piece. We of course would not be surprised that actually hearing immediately preceding phrases in the baseline timbre would facilitate making a subtle contrast with a successive timbre. Thus the data of interest comes from comparing imagery vs. control. In three of four tests (RT and accuracy x 2 pieces), imagery of the context did apparently facilitate the contrast judgment.

However, it is worthwhile examining the control condition before accepting this conclusion absolutely. The control condition took place before the piece was learned. It is possible that repeated listening of the piece might have sharpened the ability to discriminate that heard timbre, either the overall palette or the notes immediately preceding the target, from a filtered sound. Presenting the control condition after the learning phase is feasible if one simply asks the participant to compare the two extracts without mention of imagery or intervening music. This would be a more conclusive test of whether directed imagery of intervening music improves discrimination. Assuming imagery still shows superiority over control, then we would indeed have evidence from a new kind of indirect test of the ability of people to imagine more complex timbres than have been used previously (although not knowing the pieces in question, I do not actually know what the timbres were). However, we still must be cautious in assuming that an extended phrase of music was imagined successfully, as the task could be done by imagining a small excerpt of the piece immediately preceding the target measure.

The other way in which this study differs from the other studies in timbre imagery is that by the participants' own admission, they were unable to imagine the timbre. In almost all studies of imagery that I am familiar with, both auditory and visual, an assumption and a finding is that participants re-experience the percept in some way. In some studies they are directed to do so, in other studies, such as with mental rotation, they report with high frequency an ability to "call up" the requested information and then to operate on it. I consulted several cognitive psychology textbooks on my shelf and most defined mental imagery by referring to the representation of something similar to sensory experience. Thus I am not entirely convinced that the current participants were using "imagery" as the term is standardly used, unless we simply equate "imagery" with any memory representation. In these experiments, it is possible that participants could have coded the baseline timbre in an abstract or verbal way. Eliciting the subjective experience of imagery would be important in any future studies of timbre imagery for these more complex materials, in my opinion.

Nevertheless, the inability of many of Bailes's volunteers to imagine timbre as standardly defined raised the question of individual differences in auditory imagery ability. Crowder (1989) found that several of his participants were absolutely unable to ignore timbre (imagined or heard) in making pitch judgments. This suggests a good ability to call up images but an inability to control the process thereafter. Aleman, Nieuwenstein, Böcker, and de Haan (2000) compared musicians and nonmusicians in a musical pitch comparison task that was similar to the one Zatorre and I used (1993), a nonmusical auditory imagery task, and a visual imagery task. Musicians were superior to nonmusicians in both auditory tasks, but not the visual task, again suggesting individual differences based on training (or propensity). In my lab, I routinely either ask for two years of musical training as a criterion for entry into even simple auditory imagery studies, because I do occasionally find people seemingly incapable of generating or maintaining images. I 
also use a questionnaire asking people to self-rate vividness and ability to control mental images, and find a wide range in this self-report (and lower ratings, on average, than parallel items for visual imagery).

Thus we have hints that people may vary quite a bit on ability to image musical dimensions, and it is possible that this variability would be exaggerated in aspects more difficult to imagine. Certainly imagining anything extended in time would put stress on working memory structures, and even for brief stimuli, Pitt and Crowder (1992) showed that the dynamic element of timbre was hard to imagine. A long passage of music therefore changes in time on both a fine scale (dynamic aspects of timbre) and a coarser one (as notes and rhythms unfold). Thus it may not be surprising that consciously imagining timbre of an extended passage would be quite challenging and perhaps only accessible to those with large working memory spans, musical training, or both. This hypothesis could be tested in future work.

\section{REFERENCES}

Aleman, A., Nieuwenstein, M. R., Böcker, K. B. E. \& de Haan, E. H. F. (2000). Music training and mental imagery ability. Neuropsychologia, Vol. 38, pp. 1664-1668.

Bailes, F. (2007). Timbre as an elusive component of imagery for music. Empirical Musicology Review, Vol. 2, No. 1, pp. 17-30.

Crowder, R. G. (1989). Imagery for musical timbre. Journal of Experimental Psychology: Human Perception and Performance, Vol. 15, pp. 472-478.

Halpern, A. R., Zatorre, R. J., Bouffard, M. \& Johnson, J. (2004). Behavioral and neural correlates of perceived and imagined musical timbre. Neuropsychologia, Vol. 42, pp. 1281-1292.

Krumhansl, C. L. \& Iverson, P. (1992). Perceptual interactions between musical pitch and timbre. Journal of Experimental Psychology: Human Perception and Performance, Vol. 18, pp. 739-751.

Pitt, M. A., \& Crowder, R. G. (1992). The role of spectral and dynamic cues in imagery for musical timbre. Journal of Experimental Psychology: Human Perception and Performance, Vol. 7, pp. 28-38.

Warker, J. A. \& Halpern, A. R. (2005). Musical stem completion: Humming that note. American Journal of Psychology, Vol. 118, pp. 567-585.

Zatorre, R. J. \& Halpern, A. R. (1993). Effect of unilateral temporal-lobe excision on perception and imagery of songs. Neuropsychologia, Vol. 31, pp. 221-232. 